Alberto T. Penteado, Jan C. Schöneberger, Erik Esche, Hamid R. Godini, Günter Wozny, Jens-Uwe Repke

\title{
Sequential Flowsheet Optimization: Maximizing the Exergy Efficiency of a High-Pressure Water Scrubbing Process for Biogas Upgrade
}

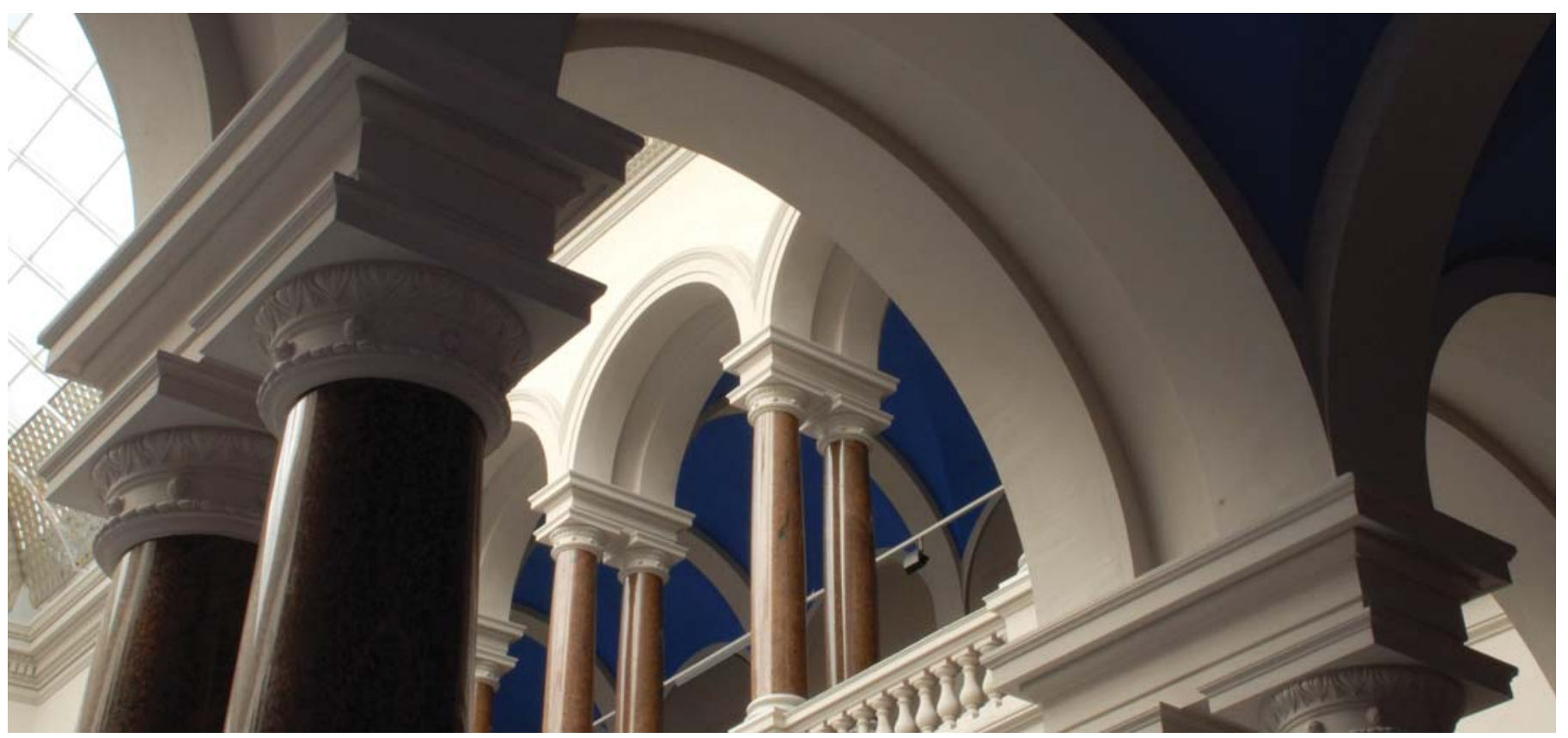

Penteado, A. T., Schöneberger, J. C., Esche, E., Godini, H. R., Wozny, G., \& Repke, J.-U. (2018).

Sequential Flowsheet Optimization: Maximizing the Exergy Efficiency of a High-Pressure Water Scrubbing Process for Biogas Upgrade. In: 28th European Symposium on Computer Aided Process Engineering. (Computer Aided Chemical Engineering ; 43). pp. 1329-1334.

https://doi.org/10.1016/b978-0-444-64235-6.50233-3 


\title{
Sequential Flowsheet Optimization: Maximizing the Exergy Efficiency of a High-Pressure Water Scrub- bing Process for Biogas Upgrade
}

\author{
Alberto T. Penteado ${ }^{a}{ }^{*}$, Jan C. Schöneberger ${ }^{b}$, Erik Esche ${ }^{a}$, Hamid R. Godini ${ }^{a}$, \\ Günter Wozny ${ }^{\mathrm{a}}$ and Jens-Uwe Repke ${ }^{\mathrm{a}}$ \\ ${ }^{\text {a }}$ Process Dynamics and Operations Group, Technische Universität Berlin, Sekretariat KWT 9 - \\ Straße des 17. Juni 135, 10623 Berlin, Germany \\ ${ }^{\mathrm{b}}$ Chemstations Europe GmbH, Lindencorso - Unter den Linden 21, 10117 Berlin, Germany \\ alberto.penteado@tu-berlin.de
}

\begin{abstract}
Biogas is an important renewable energy source and potential raw material for the chemical industry. Its utilization frequently requires a treatment and/or upgrade step. The aim here is to maximize the exergy efficiency of a high-pressure water scrubbing process for upgrading biogas into biomethane by coupling a sequential modular simulation flowsheet with different optimization algorithms. By setting adequate operating pressures, and reducing cycle water and stripping air flowrates, an exergy efficiency of $92.4 \%$ is reached.
\end{abstract}

Keywords: biogas, biomethane, high-pressure water scrubbing, sequential modular simulation, black-box optimization

\section{Introduction and Motivation}

Biogas is a mixture containing 50-75\% $\mathrm{CH}_{4}, 30-50 \% \mathrm{CO}_{2}$, as well as $\mathrm{H}_{2} \mathrm{O}, \mathrm{N}_{2}, \mathrm{O}_{2}$, and minor amounts of contaminants such as $\mathrm{H}_{2} \mathrm{~S}, \mathrm{NH}_{3}$, aromatics, and siloxanes (Rasi et al., 2007). It is produced from the anaerobic digestion of different substrates by methanogenic bacteria. It plays an important role in a sustainable energy mix because, differently from other renewables, its production does not rely solely on climatic factors. The European Union has set a target of having $20 \%$ of its 2020 energy demand supplied by renewable systems, out of which at least $25 \%$ can originate from biogas (Holm-Nielsen et al., 2009). Biogas can be utilized for electricity and heat generation within a Combined Heat and Power (CHP) plant. The methane in biogas can alternatively be converted into syngas via reforming (Chen et al., 2017) or ethylene via oxidative coupling (Penteado et al., 2017). A base treatment, consisting of cooling and drying followed by the removal or reduction of the $\mathrm{H}_{2} \mathrm{~S}$ content, is required prior to most biogas utilizations (Holm-Nielsen et al., 2009). The biogas upgrade consists in the removal of $\mathrm{CO}_{2}$ to increase the gas' calorific value to that of pipeline-quality natural gas. The resulting gas is often called biomethane and can be employed as a vehicular fuel, in fuel cells, or injected into the natural gas grid.

Different processes are available to upgrade the biogas. Sun et al. (2015) published a major review on the available methods. The selection is done on a case-by-case basis, by considering the specific raw biogas composition, amount and nature of contaminants, production scale, application requirements, and available infrastructure and utilities. The most common ones are High Pressure Water Scrubbing (HPWS), Amine Scrubbing (AMS), Pressure Swing Adsorption (PSA), Gas Permeation (GP), and Cryogenic Distillation (CD) (Sun et al., 2015). Hybrid concepts can also be 
employed to explore the main advantages of each technology (Scholz et al., 2013). The HPWS is one of the most used biogas upgrade technologies in Europe (Scheftelowitz et al., 2015). A quantitative comparison of the upgrade cost is presented in Figure 1. The calculation is done with the Biomethane Calculator (Miltner et al., 2013). Given inputs such as feed gas flow and composition; desired upgrading technology; and the required biomethane purity, the software yields methane recovery, production, investment and operating costs, and the specific biomethane production cost, thus enabling a preliminary assessment of upgrading plants. It can be seen from Figure 1, that for the gas composition and flow rate used in this study and specified in Table 1, the HPWS is very competitive. It tends to get even more competitive for larger scale plants.

A number of authors have assessed the HPWS process from an energy and economic standpoint. Rotunno et al. (2017) modeled this process in Aspen Plus using rigorous rate-based simulations to design the absorption and stripping columns. Budzianowski et al. (2017) also developed non-equilibrium Matlab models for a number of different configurations of HPWS and Near Atmospheric Pressure Water Scrubbing (NAPWS), using plant data for model validation, and finally estimating the energy requirement for the upgrade process in each configuration. The study concludes that using high pressure absorption, intermediary flash for methane recovery, and stripping for water regeneration provides an efficient solution. Wylock and Budzianowski (2017) later extend the previous contribution considering only the scrubber-flash-stripper configuration and focusing on the sizing of the column packing height, and further performance estimation for the system. While all of these studies perform the sizing and rating of the absorption and stripping columns using rigorous rate-based models, the operating conditions are determined by heuristics, experience, or by carrying out extensive simulations and sensitivity studies with one parameter being varied at the time, potentially leading to sub-optimal results. In contrast, the present study aims at determining the operating conditions for a HPWS process by means of mathematical optimization. The objective is to maximize the process' exergy efficiency given 8 continuous and bounded decision variables subject to product specification constraints. To this purpose, a framework for the optimization of sequential modular simulations is developed.

\section{Methods}

A HPWS process using a scrubber-flash-stripper configuration is modeled in the software Chemcad7. The simulation flowsheet is presented in Figure 2. The raw biogas (stream 1) is pressurized in a three stage compression section (equipment 1,20 and 4) with intermediate cooling (equipment $2,21,5$ ) in the upstream of the absorption column or scrubber (equipment 6 ). In the scrubber, the biogas is contacted with pressurized water fed to the top of the column. The gases dissolve in water, allowing the removal of the $\mathrm{CO}_{2}$ and $\mathrm{H}_{2} \mathrm{~S}$, but also causing some $\mathrm{CH}_{4}$ losses. The biomethane (stream 8), leaves the column at the top. The bottoms (stream 9) is then flashed (equipment 8) to an intermediate pressure in order to recover some $\mathrm{CH}_{4}$, which is recycled back to an intermediate compression stage. The flash's liquid (stream 12) enters the second column, where $\mathrm{CO}_{2}$ and $\mathrm{H}_{2} \mathrm{~S}$ are stripped out with air (stream 13), which is fed via a blower (equipment 10). The stripper (equipment 9 ) is operated at a pressure of 1.1 bar. The regenerated water is partially purged 
(stream 18), mixed with fresh water (stream 20), pumped to the absorption pressure (equipment 17), and passes through a heat exchanger (equipment 18) before being recirculated back to the absorption column. The gas stream leaving the stripper column passes a bio-filter (equipment 19) that removes the $\mathrm{H}_{2} \mathrm{~S}$ before leaving the process e.g. through a stack. The 8 continuous decision variables to be determined are the two intermediary pressures in the compression, the absorption pressure, the temperature of the gas and the liquid fed to the absorber, the cycle water and stripping air flowrates, and the cycle water purge ratio. Their initial values and bounds are listed in Table 2 .

\subsection{Modeling}

The Volume-Translated Peng-Robinson Equation of State is used to model the system, allowing for consistent physical and chemical exergy calculations in both liquid and gas phases in all streams. The table of binary parameters is completed by using data from Schmid et al. (2014) and with Twu parameters from Dortmund Data Bank (2015). More modeling details and validations are described by Schöneberger and Fricke (2017).

A typical biogas composition, as given by Rasi et al. (2007), is assumed according to Table 1. The biomethane must contain at least $96 \mathrm{~mol} \%$ of $\mathrm{CH}_{4}$ and maximum $20 \mathrm{ppm}_{V}$ of $\mathrm{H}_{2} \mathrm{~S}$. Compressors have an adiabatic efficiency of $50 \%$ and inter-stage cooling to $5 \mathrm{~K}$

Table 1: Raw biogas feed stream

\begin{tabular}{lcl}
\hline Flow & 300 & $\mathrm{Nm}^{3} / \mathrm{h}$ \\
Temperature & 348 & $\mathrm{~K}$ \\
Pressure & 1.0 & bar \\
Composition & & \\
$\mathrm{N}_{2}$ & 0.97 & $\mathrm{~mol} \%$ \\
$\mathrm{O}_{2}$ & 0 & $\mathrm{~mol} \%$ \\
$\mathrm{CH}_{4}$ & 60 & $\mathrm{~mol} \%$ \\
$\mathrm{CO}_{2}$ & 36 & $\mathrm{~mol} \%$ \\
$\mathrm{H}_{2} \mathrm{~S}$ & 0.03 & $\mathrm{~mol} \%$ \\
$\mathrm{H}_{2} \mathrm{O}$ & 3.0 & $\mathrm{~mol} \%$ \\
\hline
\end{tabular}
above dew point. Cooling water is available at $298 \mathrm{~K}$. Cycle water make-up is provided as saturated steam at $1.1 \mathrm{bar}$. This is done to reflect the exergy consumption for the water treatment and penalize fresh-water consumption, which would otherwise have zero physical exergy. The water pump (equipment 17) has an adiabatic efficiency of $70 \%$. Stripping air is fed at $298 \mathrm{~K}, 1.013$ bar, and dry. Pressure drop in exchangers is neglected. The absorber and stripper are packed columns using Mellapack 250Y with 20 and 10 stages respectively. The use of non-equilibrium models largely increases computational effort and column flooding can cause non-convergence of the flowsheet, which are drawbacks for the application of such models within optimization. A rate-based model is, therefore used to estimate Murphree efficiencies that are then set constant in the equilibrium stage model used in the optimization.

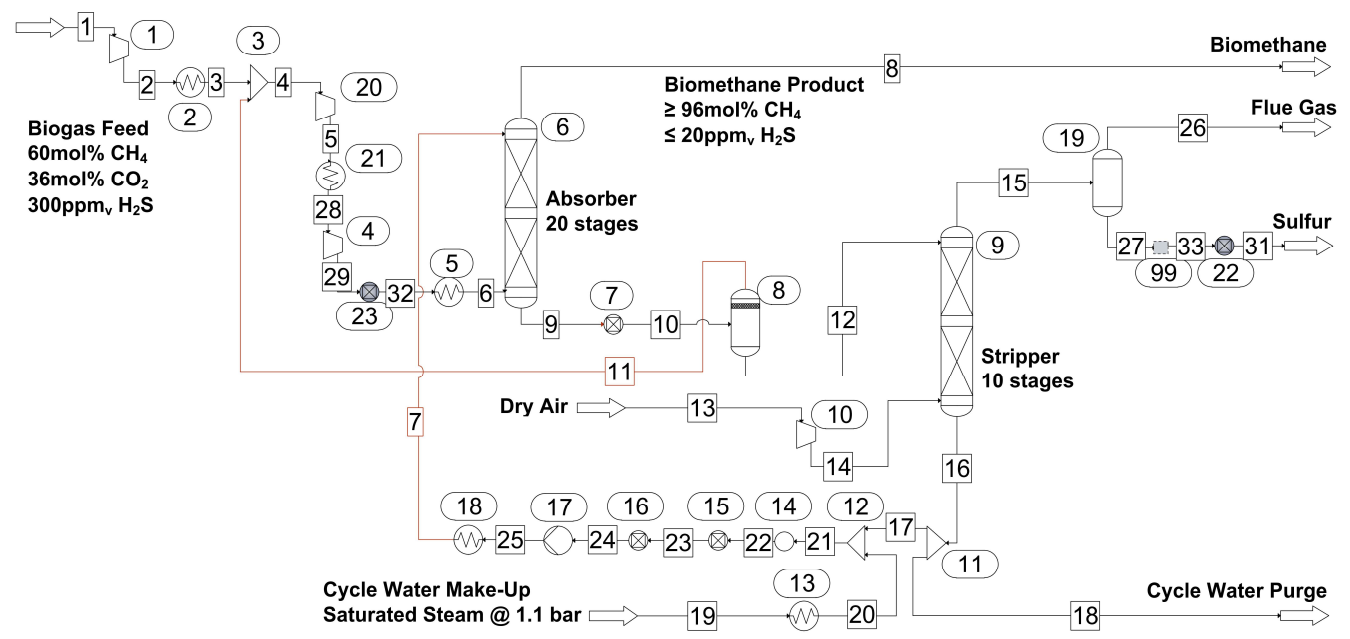

Figure 2: Simulation flowsheet of the HPWS process implemented in Chemcad7 


\subsection{Exergy Calculation}

The exergy efficiency is taken as the main objective for the optimization, as it reflects thermodynamic inefficiencies that translate into operating cost (energy consumption) and environmental impact (methane slip). The exergy rate of a stream is given as the sum of its physical and chemical exergy rates, calculated as in Equations 1 and 2 respectively. The thermodynamic environment is defined at $298 \mathrm{~K}$ and 1.0 bar. Diederichsen reference environment and ideal mixing is assumed for chemical exergies. For the work streams in the compressors and pump, the exergy rate is equal to the calculated power. The exergy efficiency, given by Equation 3, is defined as the ratio between the recovered exergy rate in the form of the biomethane product stream and all exergy fed to the process in the form of electricity to run compressors and the pump, the biogas feed stream, the stripping air, and the steam make-up for the water cycle.

$$
\begin{array}{r}
\dot{E}_{s}^{P H}=\dot{H}_{s}\left(T_{s}, p_{s}, z_{s}\right)-\dot{H}_{s}\left(T^{r e f}, p^{r e f}, z_{s}\right)-T^{r e f} \cdot\left[\dot{S}_{s}\left(T_{s}, p_{s}, z_{s}\right)-\dot{S}_{s}\left(T^{r e f}, p^{r e f}, z_{s}\right)\right] \\
\dot{E}_{s}^{C H}=\dot{n}_{s} \cdot \sum_{c=1}^{N C}\left[z_{s, c} \cdot e_{c}^{C H}+R \cdot T^{r e f} \cdot z_{s, c} \cdot \ln \left(z_{s, c}\right)\right] \\
\eta_{\text {exergy }}=\frac{\dot{E}_{8}}{\dot{W}_{1}^{e l}+\dot{W}_{20}^{e l}+\dot{W}_{4}^{e l}+\dot{W}_{10}^{e l}+\dot{W}_{17}^{e l}+\dot{E}_{1}+\dot{E}_{19}+\dot{E}_{13}}
\end{array}
$$

\section{Optimization Framework}

The aim is to maximize the value of the exergy efficiency. Constraints are set for a minimum $\mathrm{CH}_{4}$ content of $96.0 \mathrm{~mol} \%$ and a maximum $\mathrm{H}_{2} \mathrm{~S}$ content of $20 \mathrm{ppm}_{V}$ in the biomethane product gas. To solve this NLP, a framework is developed in Matlab by using the OPC Toolbox to communicate with the Chemcad7 flowsheet as a black-box simulation server, as well as different optimizers. The implementation allows for an easy and flexible objective formulation in Matlab, by reading the enthalpy and entropy rates from the Chemcad7 flowsheet, and calculating the exergy rates and then returning the negative value of the exergy efficiency to the optimizer. In a similar fashion, the constraints are evaluated inside Matlab by reading the composition of the product stream. To avoid additional unnecessary time-consuming simulations, values are saved in variables accessible by both functions. With a single black-box simulation, it is possible to evaluate the objective and the constraints. The same is true for numerical evaluation of gradients and jacobians, if required by the optimizer.

Black-box optimization with sequential modular flowhseets is difficult because the problem structure typically causes the objective function to be discontinuous, non-differentiable, not defined in many points, noisy, and subject to relaxable and unrelaxable nonlinear constraints from the simulation environment (Martelli and Amaldi, 2014). The use of gradient-based algorithms, which are the preferred choice, can therefore become very challenging. The numerical gradients might be undefined, expensive to evaluate, or simply of no use because of poor quality. Hence, direct search methods became very popular among engineers and researchers to deal with this type of optimization (Martelli and Amaldi, 2014).

To allow for enough flexibility, both gradient-based and direct-search algorithms are implemented in the framework: Matlab's Genetic Algorithm (GA) and Particle-Swarm Optimization (PSO) solvers, and the Mesh Adaptive Direct Search (MADS) algorithm NOMAD (Le Digabel, 2011) provided in the OPTI-Toolbox (Currie and Wilson, 2012). In order to use the available Sequential Quadratic Programming (SQP) algorithm provided with Chemcad7, an additional VBA unit operation is added to the flowsheet and programmed to calculate the objective value. It is not the aim of this study to provide a rigorous solver benchmark, but rather demonstrate the flexibility and try to achieve better results by exploiting the advantages of each search method. 
Table 2: Decision variables and results

\begin{tabular}{llccccccc}
\hline \multirow{2}{*}{ Variables } & \multirow{2}{*}{ Units } & $\begin{array}{c}\text { Lower } \\
\text { Bound }\end{array}$ & $\begin{array}{c}\text { Upper } \\
\text { Bound }\end{array}$ & $\begin{array}{c}\text { Initial } \\
\text { Value }\end{array}$ & GA & PSO & MADS & SQP \\
\hline Exergy Efficiency & $\%$ & 0 & 100 & 55.48 & 90.16 & 92.34 & 92.39 & 92.40 \\
Function Evaluations & - & 0 & - & - & 6,480 & 2,053 & 530 & 461 \\
\hline Intermediate Pressure & $\mathrm{bar}$ & 1.1 & 15.0 & 2.6 & 6.91 & 6.48 & 8.30 & 7.72 \\
Intermediate Pressure 2 & $\mathrm{bar}$ & 1.1 & 20.0 & 7.5 & 13.07 & 12.53 & 11.50 & 14.48 \\
Absorber Pressure & $\mathrm{bar}$ & 1.1 & 25.0 & 17.5 & 21.42 & 21.15 & 24.87 & 25.00 \\
Liquid Feed Temperature & $\mathrm{K}$ & 303 & 353 & 305 & 332 & 303 & 303 & 303 \\
Gas Feed Temperature & $\mathrm{K}$ & 303 & 353 & 323 & 328 & 351 & 353 & 303 \\
Cycle Water Flowrate & $\mathrm{Nm}^{3} / \mathrm{h}$ & 5 & 500 & 236 & 29.19 & 30.61 & 25.21 & 25.92 \\
Stripping Air Flowrate & $\mathrm{Nm}^{3} / \mathrm{h}$ & 10 & 1000 & 850 & 690.92 & 35.54 & 39.14 & 42.21 \\
Purge Ratio & - & 0.01 & 0.1 & 0.03 & 0.017 & 0.01 & 0.01 & 0.01 \\
\hline
\end{tabular}

\section{Results}

The optimal value for the purge ratio is found to be at its lower bound. Even though a smaller purge requires a higher water recirculation rate and more pump duty, the effect of the reduced fresh water requirement and its associated exergy is much more pronounced. As expected, the liquid feed temperature is also at its lower bound. The gas feed temperature has little influence to the efficiency. The intermediate pressure 2 can be almost independently optimized. However, the intermediary/flash pressure, absorption pressure, cycle water flow rate, and stripping air flow rates are highly coupled and many points with high exergy efficiencies, i.e. $\geqslant 90 \%$, have been found at fairly different conditions as intermediary results. A higher absorption pressure increases the compression duty, but reduces the required cycle water and stripping air flow rates to keep the product purity constraints fulfilled.

In this study, a rational exergy efficiency of $92.40 \%$ has been reached. A large reduction in cycle water and stripping air flow rates could be achieved, while keeping the product within specification. Although not exactly an exergy efficiency, Rotunno et al. (2017) applied a conversion factor of 0.41 to transform primary energy into electricity and then calculated an efficiency of $87.3 \%$ for a HPWS process using the same configuration. The results of this study are, thus in the same order of magnitude from previously published results. The optimal absorption pressure is found to be in its upper bound and above the initial point of 17.5 bar and above the range of 7-12 bar found as typical values in other literature cases such as (Budzianowski et al., 2017), (Wylock and Budzianowski, 2017), and (Rotunno et al., 2017). The reason is probably that considering the water make-up stream to be saturated steam over-penalizes the water usage and leads to an operating point at a higher pressure and with lower water recirculation and fresh water consumption. Nevertheless, if the water make-up stream would be liquid at ambient conditions, it would have zero physical exergy and lead to excessive use of water. To avoid this, a different objective function, e.g. operating cost, can be used in future studies.

The SQP algorithm is the most efficient and effective of the tested solvers, reaching a stationary point within 47 iterations and 461 function evaluations. The gradients are built by central finite differences with the default step size of 0.005 . It is essential to keep the step size long enough compared to the simulation tolerance. Most numeric gradient methods used in optimization have a way lower step size by default, e.g. $10^{-6}$, while tear-stream tolerances in sequential simulations is often $10^{-3}$ or $10^{-4}$. The solver NOMAD with orthogonal polling directions provided great usability with significant objective improvement with its parameters left to default. For GA and PSO, a way larger number of function evaluations is required to reach similar objective values. 


\section{Conclusion}

In this contribution, the operating conditions of a high-pressure water scrubbing process using a scrubber-flash-stripper configuration is determined by mathematical optimization. An operating point with $92.40 \%$ exergy efficiency is achieved using a SQP algorithm. A flexible framework for the optimization of sequential modular simulations is developed and presented. Future works include extending its usability to handle larger and more complex flowsheets with integer and binary decisions. Parallelization must also be addressed given the speed-up potential.

\section{Acknowledgement}

Alberto T. Penteado acknowledges the funding from CAPES / Brazil (11946/13-0). Financial support from the Cluster of Excellence Unifying Concepts in Catalysis by the German Research Foundation (DFG EXC 314) and from the German Federal Ministry of Education and Research (BMBF 10044119) are also gratefully acknowledged.

\section{References}

W. M. Budzianowski, C. E. Wylock, P. A. Marciniak, 2017. Power requirements of biogas upgrading by water scrubbing and biomethane compression: Comparative analysis of various plant configurations. Energy Conversion and Management 141, 2-19.

X. Chen, J. Jiang, K. Li, S. Tian, F. Yan, 2017. Energy-efficient biogas reforming process to produce syngas: The enhanced methane conversion by o2. Applied Energy 185, 687-697.

J. Currie, D. I. Wilson, 2012. Opti: Lowering the barrier between open source optimizers and the industrial matlab user. In: N. Sahinidis, J. Pinto (Eds.), Foundations of Computer-Aided Process Operations. Savannah, Georgia, USA.

Dortmund Data Bank, 2015. Ddb explorer version 2015. URL www.ddbst.com/free-software.html

J. B. Holm-Nielsen, T. Al Seadi, P. Oleskowicz-Popiel, 2009. The future of anaerobic digestion and biogas utilization. Bioresource technology 100 (22), 5478-5484.

S. Le Digabel, 2011. Algorithm 909. ACM Transactions on Mathematical Software 37 (4), 1-15.

E. Martelli, E. Amaldi, 2014. Pgs-com: A hybrid method for constrained non-smooth black-box optimization problems. brief review, novel algorithm and comparative evaluation. Computers and Chemical Engineering 63, 108-139.

M. Miltner, A. Makaruk, M. Harasek, Oct. 2013. Biomethane calculator version 2.1.1. Software. URL bio.methan. at

A. T. Penteado, M. Kim, H. R. Godini, E. Esche, J.-U. Repke, 2017. Biogas as a renewable feedstock for green ethylene production via oxidative coupling of methane: Preliminary feasibility study. In: Chemical Engineering Transactions. Vol. 61. The Italian Association of Chemical Engineering, pp. 589-594.

S. Rasi, A. Veijanen, J. Rintala, 2007. Trace compounds of biogas from different biogas production plants. Energy 32 (8), 1375-1380.

P. Rotunno, A. Lanzini, P. Leone, 2017. Energy and economic analysis of a water scrubbing based biogas upgrading process for biomethane injection into the gas grid or use as transportation fuel. Renewable Energy 102, 417-432.

M. Scheftelowitz, N. Rendsberg, V. Denysenko, J. Daniel-Gromke, W. Stinner, K. Hillebrand, K. Naumann, D. Peetz, C. Hennig, Thrän D., M. Beil, J. Kasten, L. Vogel, 2015. Stromerzeugung aus biomasse (vorhaben iia biomasse): Zwischenbericht mai 2015.

B. Schmid, A. Schedemann, J. Gmehling, 2014. Extension of the vtpr group contribution equation of state: Group interaction parameters for additional 192 group combinations and typical results. Industrial \& Engineering Chemistry Research 53 (8), 3393-3405.

M. Scholz, B. Frank, F. Stockmeier, S. Falß, M. Wessling, 2013. Techno-economic analysis of hybrid processes for biogas upgrading. Industrial \& Engineering Chemistry Research 52 (47), 16929-16938.

J. Schöneberger, A. Fricke, 2017. Optimizing the exergetic efficiency of a pressurized water process for bio gas cleaning. Chemical Engineering Transactions 57, 457-462.

Q. Sun, H. Li, J. Yan, L. Liu, Z. Yu, X. Yu, 2015. Selection of appropriate biogas upgrading technology-a review of biogas cleaning, upgrading and utilisation. Renewable and Sustainable Energy Reviews 51, 521-532.

C. E. Wylock, W. M. Budzianowski, 2017. Performance evaluation of biogas upgrading by pressurized water scrubbing via modelling and simulation. Chemical Engineering Science. 